\title{
Contrast effects accompanying shifts in sucrose concentration during the acquisition of a brightness discrimination
}

\author{
JOHN N. MOORE and ROBERT ADAMSON \\ Florida Atlantic University, Boca Raton, Florida 33432
}

\begin{abstract}
Twenty-eight rats were randomly assigned to four groups representing a factorial combination of two levels of preshift and two levels of postshift sucrose concentration. Following 220 preshift trials, the subjects were required to learn a brightness discrimination, and they either shifted to a novel reward concentration or maintained on the preshift magnitude. The results of an analysis of response speed indicated significant magnitude effects as well as NCE but no indication of PCE. Analysis of the number of errors indicated NCE but not PCE. The results were discussed with reference to perceptual and sequential theories of contrast effects.
\end{abstract}

Successive contrast effects, which typically accompany shifts in reward magnitude, have been the source of considerable theoretical and empirical interest since Crespi's (1942) original study. A positive contrast effect (PCE) is said to occur when subjects that are trained on small reward and subsequently shifted to large reward demonstrate enhanced performance relative to that of a constant large reward comparison condition. A negative contrast effect (NCE) is obtained when subjects that are previously trained on large reward and shifted to small reward show a depressed level of performance when compared to a group consistently reinforced with the small reward magnitude.

Two competing theoretical positions which may account for the contrast phenomena involve sequential variables (Capaldi, 1967) and perceptual variables (Bevan \& Adamson, 1960). The perceptual interpretation, based upon the notion of adaptation level (Helson, 1964), differentiates perceived reward magnitude from physical reward magnitude. Ultimately, contrast effects are generated by a discrepancy between postshift reward magnitude and the reward magnitude adaptation level that was formed during the preshift training period. If this discrepancy is negative (i.e., postshift magnitude being smaller than preshift adaptation level), NCE will be obtained. Conversely, if the discrepancy is in a positive direction (postshift magnitude larger than the preshift magnitude adaptation level) PCE will result.

The stimulus specificity or sequential position

This research was supported by a grant to the second author from the Florida Atlantic University Research Committee. John N. Moore's present address is: Southern Illinois University, Carbondale, Illinois 62901. Requests for reprints should be addressed to Dr. Robert Adamson, Florida Atlantic University, Boca Raton. Florida 33432. considers the NCE to be an instance of generalization decrement. The stimulus aftereffects of large reward $(\mathrm{SL})$ are conditioned to the instrumental response during preshift training. The stimuli present in postshift $(\mathrm{SS})$, following reduction to the small reward magnitude have but generalized ability to evoke the instrumental response, thus resulting in NCE. The sequential position accounts for the occurrence of the NCE as well as the usual finding of an absence of the PCE (Dunham, 1968), while the perceptual position demands both PCE and NCE.

An instrumental task that could serve to test between these two positions involves the choice behavior of shifted and nonshifted groups in a discrimination problem. Although neither theory specifically addresses itself to choice behavior, it might be inferred that if subjects are pretrained on a particular reward magnitude and subsequently shifted to a different magnitude prior to the acquisition of a discrimination, the shifted conditions would be expected, according to the perceptual theory, to demonstrate contrast effects. Animals shifted from large to small reward might commit more errors (NCE) during discrimination training than small reward control conditions. Similarly, subjects shifted from small to large reward should make fewer errors (PCE) than large reward controls.

According to the sequential position, however, shifting reward magnitudes should not result in contrast effects since the subjects have not previously been conditioned to respond to the positive stimulus prior to the reward shift.

The present study was designed to investigate the effects of abrupt, unsignaled shifts in sucrose concentration on both number of errors and response rate during the acquisition of a simultaneous brightness discrimination. 


\section{METHOD}

Subjects

Twenty-eight naive male albino rats, obtained from the Holtzman Company, Madison. Wisconsin, served as subjects in the investigation. The animals were individually housed and given free access to both food and water until the initiation of the experimental routine. Thereafter, animals were both food-and water-deprived except for a 10-min period of free access to wet mash following each experimental session. The subjects were 60 days old at the beginning of discrimination training.

\section{Apparatus}

A conventional operant conditioning chamber was used to shape the desired barpress response. The discrimination apparatus consisted of a modified operant chamber $45.5 \mathrm{~cm}$ long and $20.5 \mathrm{~cm}$ wide. The final $23 \mathrm{~cm}$ of the chamber was divided by a flat black partition. thus creating two straight alleys $23 \mathrm{~cm}$ long, $10 \mathrm{~cm}$ wide, and $12.5 \mathrm{~cm}$ high. A rear projection screen was mounted at the end of each alley upon which the stimuli were presented. Two manipuland a were positioned $1.5 \mathrm{~cm}$ from the projection screen and $3 \mathrm{~cm}$ above the floor of the chamber. One bar was placed on the left wall of the left alley while the second lever was mounted on the right wall of the right alley.

The forward section of the apparatus was $20 \mathrm{~cm}$ high and contained an LVE (Model 1527) liquid reinforcement dispenser which extended through the front wall of the chamber. The dispenser was centered $1.5 \mathrm{~cm}$ above the grid bar floor. The front wall of the apparatus was black Plexiglas; the remainder of the chamber was constructed from translucent Plexiglas.

A Kodak Ektagraphic slide projector. located approximately $45 \mathrm{~cm}$ from the rear screens was used to project the stimuli. The stimuli were made by occluding either the right or left halves of two translucent $35-\mathrm{mm}$ slides. Solid state and electromechanical recording equipment was used to program slide advance and reverse as well as the total number of trials, errors, and the amount of time required to complete 10 trials. The apparatus was placed in a darkened room and a $70-\mathrm{dB}$ masking noise served to attenuate extraneous noise.

\section{Procedure}

The groups were labeled according to the percent concentration of the sucrose reward received during the preshift (preadaptation) and postshift (discrimination) phases, Groups 4-4, 32-32, 4-32, and 32-4. Reinforcement solutions were mixed by weight from tap water and commercial cane sugar.

Subjects were first barpress trained in the operant chamber and given daily practice sessions. $15 \mathrm{~min}$ in duration, for 5 consecutive days. During this period, all animals received water reinforcement.
All groups were then preadapted in the discrimination apparatus on a continuous reinforcement schedule for 4 days. The preadaptation contingency proceeded as follows: Day 1: All animals were required to make 10 forced choice responses in the right alley; Day 2: Subjects made 10 responses in the left alley; Days 3 and 4: Subjects made 50 responses in each alley on both days.

The animals received a total of 220 reinforcements, either $4 \%$ or $32 \%$ sucrose, during the preadaptation phase. The projection lamp was illuminated during this stage of the experiment, but an opaque screen precluded the animals from viewing the stimuli.

The discrimination task, which consisted of a simultaneous brightness discrimination, required the subject to traverse either the right or left alley, press the manipulandum, and run to the opposite end of the chamber to secure reinforcement if a correct response was made. After every response, the projection lamp extinguished for $3 \mathrm{sec}$. This allowed a slide to advance or reverse if programmed to do so.

Discrimination training was initiated on Day 5. Subjects were weighed and either shifted to the alternate reward solution or continued to receive the same concentration obtained during preadaptation. During acquisition of the discrimination task. subjects received 200 massed trials, all administered on the same day, in which all responses in the bright alley were reinforced while all responses in the dark alley went unrewarded. The position of the positive stimulus was varied according to a predetermined random order. with each alley being correct on $50 \%$ of the trials. A correction procedure was employed to discourage position responding. This was accomplished by programming the equipment so that a stimulus slide could not advance or reverse unless a correct response was made. All response latencies were converted to speed scores.

\section{RESULTS}

The results of an analysis of subjects weights prior to discrimination training revealed an absence of signiticant differences $(F=1.16, \mathrm{df}=3 / 24, \mathrm{p}>$ .25). Figure 1, Panels A, B, and C, show appropriate comparisons for magnitude of reward effects, NCE, and PCE, respectively, over successive blocks of 40 trials, during discrimination acquisition.

Clearly, subjects learned to choose the correct alley, achieving approximately $75 \%$ correct at the end of the session. It also appears that the number of errors during acquisition was independent of reward concentrations except for a trend which indicates that
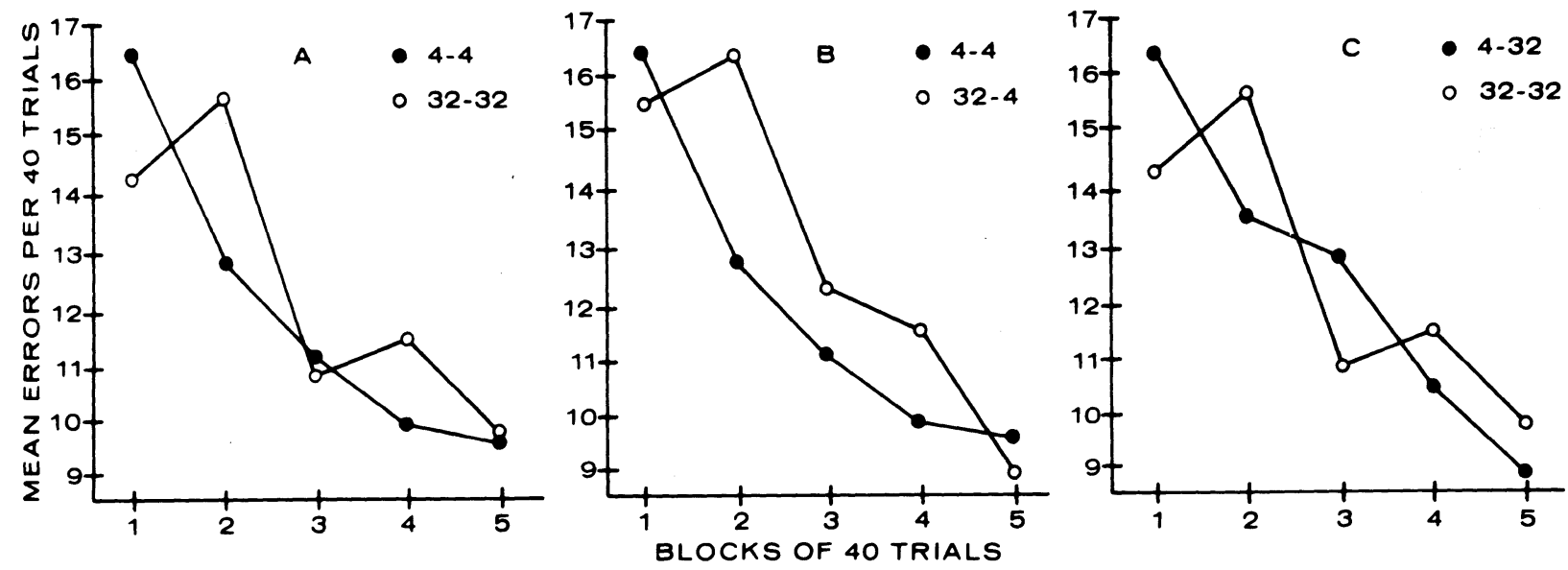

Figure 1. Mean number of errors per 40 trials. 

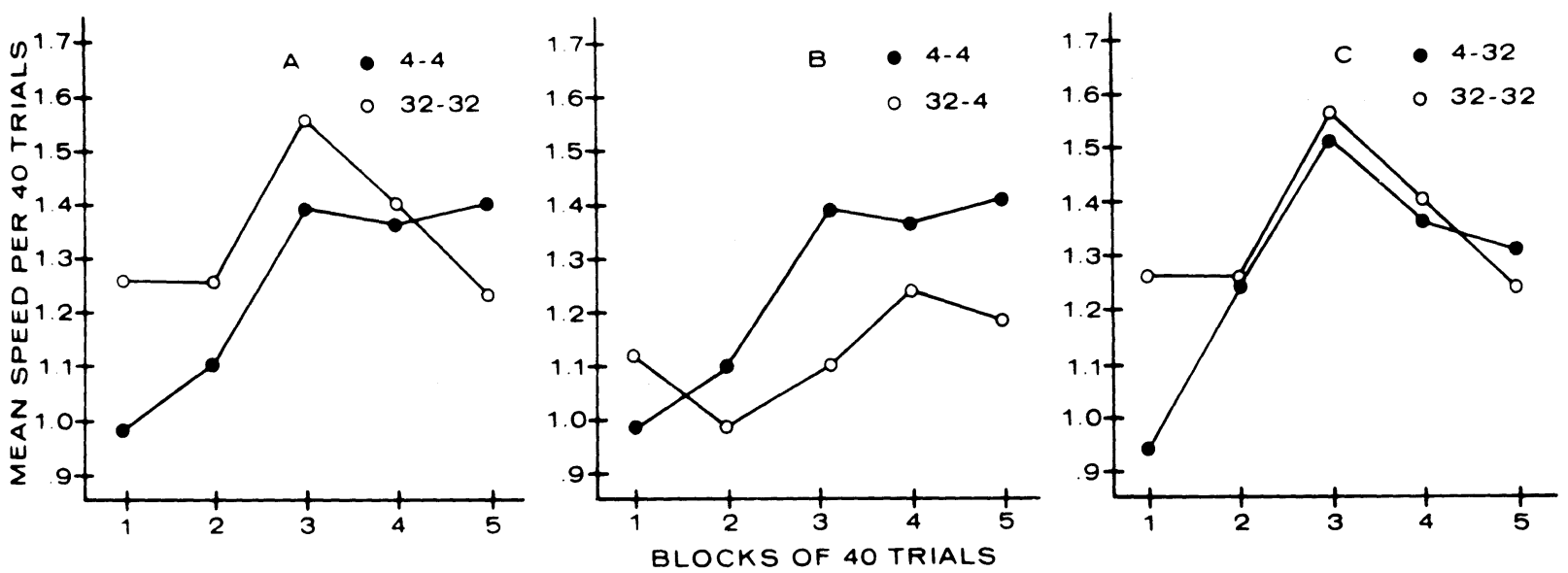

Figure 2. Mean response speed per $\mathbf{4 0}$ trials.

G32-4 made more errors than G4-4 over Blocks 2, 3, and 4 . The results of an analysis of variance (preshift magnitude by postshift magnitude by blocks) indicated a signiticant blocks effect $(F=31.96$, $\mathrm{df}=4 / 96, \mathrm{p}<.001)$ as well as a significant Preshift Magnitude by Blocks interaction effect $(F=3.22$, $\mathrm{df}=4 / 96, \mathrm{p}<.05)$. Analysis of the interaction showed that the groups that received the $32 \%$ concentration in preshift made more errors, at Block 2. than did the $4 \%$ groups $(F=9.33$, $\mathrm{df}=1,96, \mathrm{p}<.005)$. This finding was probably the result of the high variability shown by animals in G32-32. Although the Preshift by Postshift by Blocks interaction was not significant, planned comparisons, at Block 2, showed that G32-4 made more errors (NCE) than G4-4 (F=7.29, df = 1/96, p <.01). Although G32-4 continued to make more errors at Blocks 3 and 4 than G4-4, the differences were not statistically significant. No significant differences were obtained between G32-32 and G4-32.

Figure 2, Panels A, B, and C, show speed performance levels for the four groups. The figure indicates magnitude of reward effects, with G32-32 responding faster than G4-4, as well as NCE, G32-4 responding slower than G32-32, but no indication of PCE. The analysis of variance was identical to that performed on the number of errors. The results of the analysis showed only a significant blocks effect $(F=6.39$, df $=4 / 96, p<.001)$. Response speed increased over the first three blocks and then stabilized except for groups that received the $32 \%$ concentrations during postshift. Planned comparisons showed that G4-4 responded slower than did G32-32 at Block $1(\mathrm{~F}=4.2$. $\mathrm{df}=1 / 96, \mathrm{p}<.05)$. Although the trend continued until the final block of trials, none of the differences were statistically significant. Group 4-32 was also slower than G32-32 at Block 1 $(\mathrm{F}=4.80, \mathrm{df}=1 / 96, \mathrm{p}<.05)$ but quickly adjusted its performance to that of the control group. A significant NCE was also obtained at Block 3 $(F=4.2, \quad$ df $=1 / 96, \quad p<.05), \quad$ with $G 32-4$ responding slower than G4-4. Subjects in G32-4 continued to respond slower than G4-4 until the last block of trials.

\section{DISCUSSION}

One potentially important aspect of the present investigation was the finding that subjects learned the brightness discrimination in an operant choice learning situation. The apparatus and method used in the experiment should be of interest to researchers involved in discrimination learning.

Both the sequential theory as well as the perceptual model may adequately account for the observation of NCE in the response speed measure. The absence of PCE, in terms of response speed, presents a problem for the perceptual position. Bower (1961) has suggested that the usual finding of NCE and the absence of PCE may be attributable to the fact that the unshifted control conditions reach a physiological limit such that shifted subjects are incapable of responding faster than the large-magnitude control groups.

The current procedure optimized the possibility of observing PCE since the reward shift preceded the acquisition of differential responding. If a large reward magnitude "ceiling effect" did preclude the demonstration of PCE (for response speed), it clearly was not reached until Block 3. Thus G4-32 had 80 trials to demonstrate a PCE but failed to do so. It appears, then, that contrast effects found in operant situations by shifts in sucrose reward are highly similar in their asymmetry (finding NCE but not PCE) to those found in the runway following shifts in the number of food pellets.

Although rather equivocal, the NCE observed in the present study reflected by the greater number of errors made by G32.4 as compared with G4-4 is in accord with the perceptual position. During the preadaptation phase, subjects formed an adaptation level appropriate for large reward magnitude. When shifted to the smaller magnitude, the effectiveness of the postshift reward was less for experimental as compared with control conditions because of the discrepancy between postshift magnitude and the preshift magnitude adaptation level. According to this line of reasoning, however. G4-32 should have made fewer errors than G32-32. It should be noted that a physiological limit explanation for the absence of the PCE is not appropriate when measuring choice behavior.

The sequential position encounters difficulty in accounting for the NCE shown by G4-3L. In the preshift phase, the leverpress response was conditioned to the stimulus aftereffects of large reward. The stimuli present in postshift. those associated with small magnitude. 
have limited ability to evoke the instrumental response, thus resulting in NCE for the rate measure. Differential responding in the presence of the positive stimulus, however, was not initiated until the postshitt period. Thus, the preshift magnitude should have had no effect upon subsequent choice behavior.

Additional research, utilizing a more conventional discrimination procedure as well as shifts in solid food rewards, may yet clarify the effects of reward magnitude shifts upon choice behavior.

\section{REFERENCES}

Bevan. W., \& Adamson, R. Reinforcers and reinforcement: their relation to maze performance. Journal of Experimental Prichology. 1960. 59. 226-232.
BOWER, G. H. A contrast effect in differential conditioning. Journal of Experimental Psychology, 1961, 62. 196-199.

CAPALDI. E. J. A sequential hypothesis of instrumental learning. In K. W. Spence \& J. T. SPENCE (Eds.), The psychology of learning and motivation (Vol. 1). New York: Academic Press, 1967. Pp. 67-156.

CRESPI, L. P. Quantitative variation of incentive and performance in the white rat. American Journal of Psychology, 1942. 55. 467-517.

Dunham, P. J. Contrasted conditions of reinforcement: A selective critique. Psychological Bulletir, 1968, 69, 295-315.

Helson. H. Adaptation-level theory: An experimental and systematic approach to behavior. New York: Harper and Row. 1964.

(Received for publication January 10. 1976.) 